\title{
Full-Body Radiographic Analysis of Postoperative Deviations From Age-Adjusted Alignment Goals in Adult Spinal Deformity Correction and Related Compensatory Recruitment
}

\author{
PETER G. PASSIAS, MD,${ }^{1}$ CYRUS M. JALAI, BA, ${ }^{1}$ BASSEL G. DIEBO, MD ${ }^{2}$ DANA L. CRUZ, MD,${ }^{1}$ \\ GREGORY W. POORMAN, BA, ${ }^{1}$ AARON J. BUCKLAND, MBBS FRACS, ${ }^{1}$ LOUIS M. DAY, BS, ${ }^{1}$ \\ SAMANTHA R. HORN, BA, ${ }^{1}$ BARTHÉLEMY LIABAUD $\mathrm{MD},{ }^{2}$ RENAUD LAFAGE, MS,${ }^{2}$ ALEXANDRA \\ SOROCEANU, MD, ${ }^{3}$ JOSEPH F. BAKER, MCH FRCSI, ${ }^{1}$ SHEARWOOD MCCLELLAND III, MD, ${ }^{1}$ \\ JONATHAN H. OREN, MD ${ }^{1}$ THOMAS J. ERRICO, MD ${ }^{1}$ FRANK J. SCHWAB, MD ${ }^{2}$ VIRGINIE LAFAGE, \\ $\mathrm{PHD}^{2}$ \\ ${ }^{1}$ Department of Orthopaedic Surgery, Hospital for Joint Diseases, NYU Langone Medical Center, New York, New York, ${ }^{2}$ Department of Orthopaedic Surgery, \\ Hospital for Special Surgery, New York, New York, ${ }^{3}$ Department of Orthopaedic Surgery, University of Calgary, Calgary, Alberta, Canada
}

\begin{abstract}
Background: Full-body stereographs for adult spinal deformity (ASD) have enhanced global deformity and lower-limb compensation associations. The advent of age-adjusted goals for classic ASD parameters (sagittal vertical axis, pelvic tilt, spino-pelvic mismatch [PI-LL]) has enabled individualized evaluation of successful versus failed realignment, though these remain to be radiographically assessed postoperatively. This study analyzes pre- and postoperative sagittal alignment to quantify patient-specific correction against age-adjusted goals, and presents differences in compensation in patients whose postoperative profile deviates from targets.

Methods: Single-center retrospective review of ASD patients $\geq 18$ years with biplanar full-body stereographic xrays. Inclusion: $\geq 4$ levels fused, complete baseline and early ( $\leq 6$-month) follow-up imaging. Correction groups generated at postoperative visit for actual alignment compared to age-adjusted ideal values for pelvic tilt, PI-LL, and sagittal vertical axis derived from clinically relevant formulas. Patients that matched exact \pm 10 -year threshold for ageadjusted targets were compared to unmatched cases (undercorrected or overcorrected). Comparison of spinal alignment and compensatory mechanisms (thoracic kyphosis, hip extension, knee flexion, ankle flexion, pelvic shift) across correction groups were performed with ANOVA and paired $t$ tests.

Results: The sagittal vertical axis, pelvic tilt, and PI-LL of 122 patients improved at early postoperative visits $(P<.001)$. Of lower-extremity parameters, knee flexion and pelvic shift improved $(P<.001)$, but hip extension and ankle flexion were similar $(P>.170)$; global sagittal angle decreased overall, reflecting global postoperative correction $\left(8.3^{\circ}\right.$ versus $\left.4.4^{\circ}, P<.001\right)$. Rates of undercorrection to age-adjusted targets for each spino-pelvic parameter were $30.3 \%$ (sagittal vertical axis), $41.0 \%$ (pelvic tilt), and $43.6 \%$ (PI-LL). Compared to matched/overcorrections, undercorrections recruited increased posterior pelvic shift to compensate $(P<.001)$; knee flexion was recruited in undercorrections for sagittal vertical axis and pelvic tilt; thoracic hypokyphosis was observed in PI-LL undercorrections. All undercorrected groups displayed consequentially larger global sagittal angle $(P<.001)$.

Conclusions: Global alignment cohort improvements were observed, and when comparing actual to age-adjusted alignment, undercorrections recruited pelvic and lower-limb flexion to compensate.
\end{abstract}

Level of Evidence: 3

Biomechanics

Keywords: adult spinal deformity, age-adjusted alignment, compensatory recruitment, full-body radiographs, global spinal alignment

\section{INTRODUCTION}

Correspondence between key radiographic parameters - sagittal vertical axis (SVA), pelvic tilt (PT), lumbo-pelvic mismatch (PI-LL) — and disabil- ity measures has resulted in advancements in complex adult spinal deformity (ASD) assessments; optimization of these parameters has consequently resulted in meaningful clinical improvements. ${ }^{1-6}$ These relationships have standardized clinical tar- 
gets to guide surgical decision making, as prescribed by the Scoliosis Research Society-Schwab ASD classification. ${ }^{7}$ Recent studies, however, have demonstrated that pathophysiological factors of age, bone mineral density, and comorbidity status mitigate the generalized applicability of predetermined realignment targets to individual deformities. Instead of one surgical solution for all, a tailored approach specified to a single patient for ideal alignment may be more appropriate for successful correction. $^{8}$

Execution of preoperative plans requires a thorough understanding of a patient's sagittal profile in conjunction with factors contributing to progressive malalignment. Lafage et $\mathrm{al}^{8}$ have emphasized age as a necessary metric to incorporate into planning to redefine alignment thresholds. Historically, ASD correction is associated with a high failure rate of around $22 \%$, despite intraoperative imaging and surgical technique improvements. ${ }^{9,10}$ Root cause analysis highlights greater baseline spino-pelvic deformity and requirements for more aggressive procedures to match patients' sagittal profiles as driving radiological failures. Consequently, suboptimal alignment outcomes are commonly attributed to deficiencies in preoperative planning strategies; prospective implementation of novel age-adjusted targets for individualized realignment may remedy this.

Intolerance to sagittal plane under- or overcorrection may result in important radiographic and clinical deteriorations, but recruitment of lower extremity musculoskeletal mechanisms to counteract postoperative malalignment remains unexplored. ${ }^{11-13}$ Advances in ASD assessment through novel full-body imaging technology accentuate the need for head-to-toe radiographic evaluation of lower extremity compensatory mechanism visualization, including knee and ankle flexion, hip extension, and pelvic displacement. Preliminary analyses have unveiled important associations between lower limb recruitment, maintenance of standing posture, and demographic characteristics, including age; such findings may precipitate changes in operative planning and realignment goals. ${ }^{14-16}$

These reports, however, only present a baseline snapshot of a patient's global alignment, as no study to date has offered a full-body analysis following surgical sagittal deformity correction using new agealignment goals as indicative of successful versus failed realignment. This study thus analyzes pre- and postoperative full-body alignment following spinal deformity correction to evaluate adoption of compensation in patients whose postoperative sagittal profile deviates from novel age-adjusted alignment targets.

\section{METHODS}

\section{Data Source}

This is a retrospective radiographic analysis without clinical correlation of patients visiting a single academic center for spine-related complaints from 2013 to 2015 following Institutional Review Board approval. Inclusion criteria were adults $(\geq 18$ years) undergoing $\geq 4$ levels of fusion for sagittal spinal deformity (scoliosis, kyphosis) with biplanar full-body stereotactic radiographs (EOS imaging) at baseline visit and early follow-up ( $\leq 6$ months). ${ }^{17}$ Exclusion criteria were patients with fractures, malignancies, infections, pseudarthrosis, hardware failure, or nonidiopathic or nondegenerative deformity etiologies. A subanalysis was performed on the portion of the included cohort with complete radiographic follow-up at 1 year postoperative.

\section{Data Collection and Radiographic Evaluation}

Demographic data comprised age, body mass index (BMI), and gender. Surgical variables included upper and lower instrumented vertebrae (UIV/ LIV), and osteotomy use (Smith-Petersen osteotomy, 3-column osteotomy [3CO]).

Full-body images were measured for the 122 patients who met the inclusion criteria (Surgimap, Nemaris Inc, New York, New York). ${ }^{18,19}$ Spinopelvic parameters included SVA, PT, pelvic incidence (PI), PI-LL, and thoracic kyphosis (TK) (Figure 1). Lower-limb parameters included knee flexion angle (KA: angle between the mechanical axes of the femur and tibia), ankle flexion angle (AA: angle between the mechanical axis of the tibia and the vertical), posterior pelvic shift (PS: offset between the posterosuperior aspect of the sacrum and the distal tibia anterior cortex), hip extension (SFA: angle formed by the line from the middle of S1 endplate to the midpoint of the 2 femoral heads and the line between the midpoint of the 2 femoral heads and the femoral axis), and global sagittal angle (GSA: angle subtended by a line from midpoint of $\mathrm{C} 7$ vertebra to midpoint of femoral condyles and a line from this point to the posterosuperior S1 corner). ${ }^{16,20,21}$ 


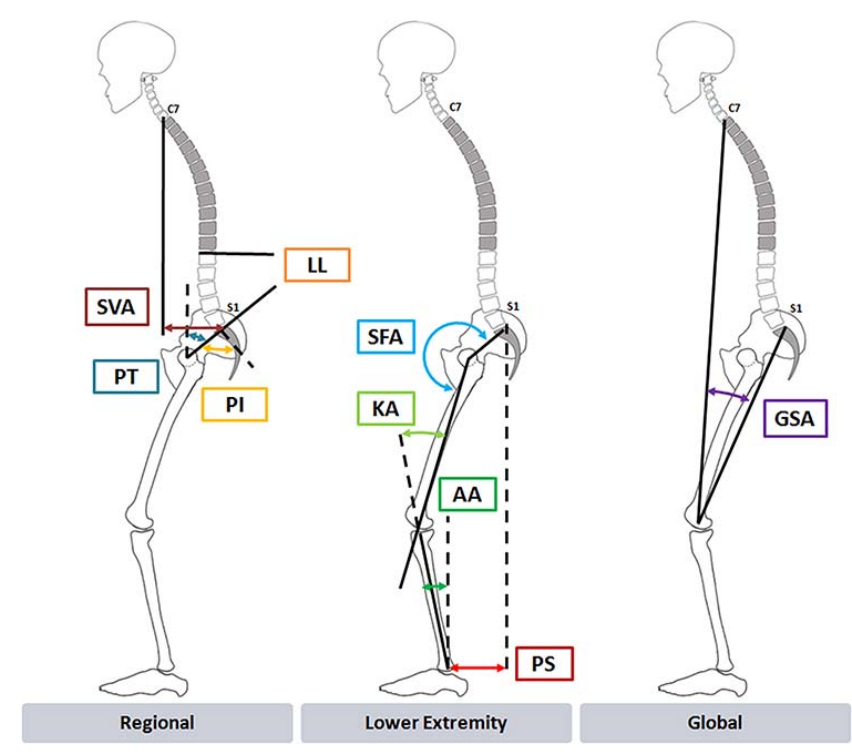

Figure 1. Measurements of regional (left), lower-limb (middle), and global (right) spinal radiographic parameters. Abbreviations: SVA, sagittal vertical axis; PT, pelvic tilt; PI, pelvic incidence; LL, lumbar lordosis; SFA, sacrofemoral angle; KA, knee angle; AA, ankle angle; PS, posterior pelvic shift; and GSA, global sagittal angle.

\section{Patient Age-Alignment Grouping}

Age-specific alignment values for SVA, PT, and PI-LL were generated for each individual patient according to previously published formulas: ${ }^{8}$

$$
\begin{gathered}
\mathrm{PI}-\mathrm{LL}=\frac{(\text { Age }-55)}{2}+3 \\
\mathrm{PT}=\frac{(\text { Age }-55)}{3}+20 \\
\mathrm{SVA}=2 \times(\text { Age }-55)+25
\end{gathered}
$$

Correction groups were then generated according to the agreement between actual measured alignment recorded at the early postoperative visit and calculated age ideals: matched, undercorrected, and overcorrected. Matched patients' actual postoperative alignment reached a \pm 10 -year interval of age-adjusted values, based on a validated method of gauging under- versus overcorrection. ${ }^{8}$ Undercorrected and overcorrected patients were aligned to targets that were $>10$ years or $<10$ years of their age in each spino-pelvic category (Figure 2).

\section{Statistical Analysis}

Descriptive statistics were used to describe categorical and continuous variables. Comparisons between baseline and postoperative alignment for

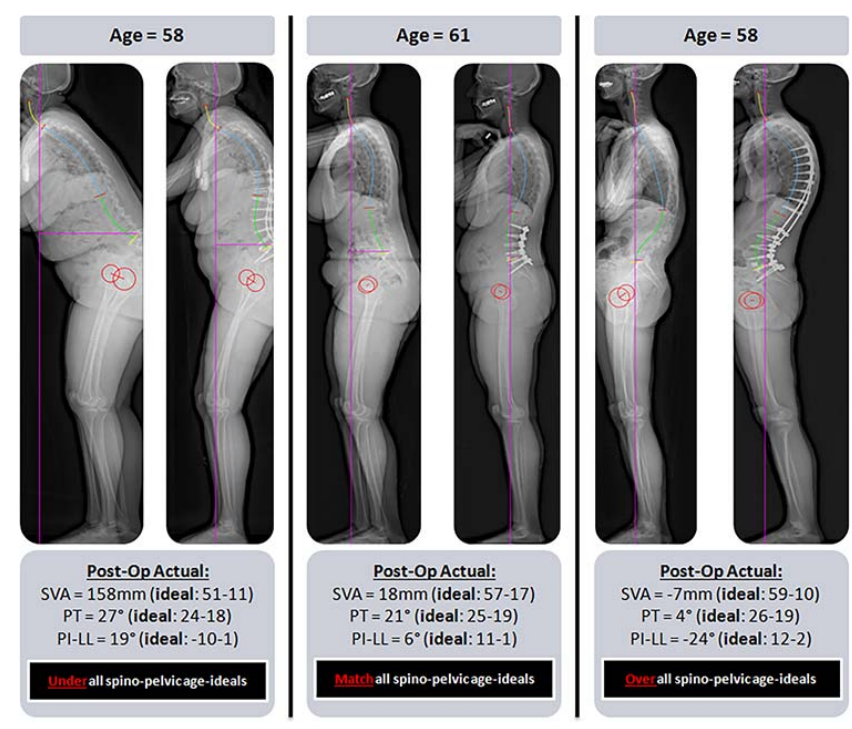

Figure 2. Case examples (baseline and early $[\leq 6$-month] postoperative) fullbody stereoradiographs of 3 patients whose actual postoperative alignment was Under (undercorrected), Match (matched), or Over (overcorrected) compared to the age-adjusted ideal for sagittal spino-pelvic parameters. Postoperative ageadjusted ideal measurement ranges for each spino-pelvic parameter are provided for a \pm 10 -year threshold range for optimal alignment based on the individual patient's age. Abbreviations: SVA, sagittal vertical axis; PI-LL, mismatch between pelvic incidence and lumbar lordosis; and PT, pelvic tilt.

compensatory mechanisms (TK, SFA, KA, AA, PS) and spino-pelvic measures (SVA, PT, PI-LL) were made between 3 correction groups with ANOVA. Statistics were performed with SPSS software (version 21.0, Armonk, New York) with statistical significance set at $P<.05$.

\section{RESULTS}

\section{Study Sample and Surgical Summary}

A total of 122 patients met inclusion criteria (mean age: $61.7 \pm 14.6$ years; mean BMI: $26.9 \pm 5.8 \mathrm{~kg} / \mathrm{m}^{2} ; 64.8 \%$ female). A mean 10.0 levels were fused among all patients. The $3 \mathrm{CO}$ rate was $14.8 \%$, and the Smith-Petersen osteotomy rate was $47.5 \%$.

\section{Radiographic Realignment Outcomes}

The average preoperative spino-pelvic parameters for all patients revealed severe baseline sagittal malalignment: $\mathrm{SVA}=73.1 \pm 73.8 \mathrm{~mm}$, $\mathrm{PT}=27.1^{\circ} \pm 12.0^{\circ}, \mathrm{PI}-\mathrm{LL}=20.5^{\circ} \pm 21.8^{\circ}$ (Table 1). The mean calculated age-ideal alignment values were: $\mathrm{SVA}=38.3 \pm 29.2 \mathrm{~mm}, \mathrm{PT}=22.2^{\circ} \pm 4.9^{\circ}$, $\mathrm{PI}-\mathrm{LL}=9.5^{\circ} \pm 17.7^{\circ}$. Compared to theoretical age-adjusted targets, actual postoperative values for all parameters were significantly larger $(P<.001)$. However, all patients significantly 
Table 1. Mean baseline, age-adjusted, and actual postoperative sagittal alignment parameter values for the entire study cohort.

\begin{tabular}{|c|c|c|c|c|}
\hline & Baseline & Ideal & Postoperative & $\boldsymbol{P}$ \\
\hline SVA (mm) & $73.1 \pm 73.8$ & $38.3 \pm 29.2$ & $35.8 \pm 51.8$ & $<.001^{\mathrm{ab}}$ \\
\hline $\mathrm{PT}\left({ }^{\circ}\right)$ & $27.1 \pm 12.0$ & $22.2 \pm 4.9$ & $24.0 \pm 10.9$ & $<.001^{\mathrm{ab}}$ \\
\hline PI-LL $\left(^{\circ}\right)$ & $20.5 \pm 21.8$ & $6.3 \pm 7.3$ & $9.5 \pm 17.7$ & $<.001^{\mathrm{ab}}$ \\
\hline $\mathrm{TK}\left({ }^{\circ}\right)$ & $38.5 \pm 19.9$ & $\ldots$ & $45.1 \pm 17.2$ & $<.001^{\mathrm{a}}$ \\
\hline $\mathrm{SS}\left({ }^{\circ}\right)$ & $28.6 \pm 13.2$ & .. & $32.8 \pm 11.4$ & $<.001^{\mathrm{a}}$ \\
\hline $\operatorname{SFA}^{\circ}\left(^{\circ}\right)$ & $204.6 \pm 9.5$ & & $204.2 \pm 10.2$ & .577 \\
\hline $\mathrm{KA}\left(^{\circ}\right)$ & $9.8 \pm 9.2$ & $\ldots$ & $6.8 \pm 8.4$ & $<.001^{\mathrm{a}}$ \\
\hline $\mathrm{AA}\left({ }^{\circ}\right)$ & $7.8 \pm 4.0$ & .. & $7.4 \pm 4.5$ & .170 \\
\hline PS (mm) & $38.8 \pm 47.8$ & . & $16.7 \pm 38.7$ & $<.001^{\mathrm{a}}$ \\
\hline $\operatorname{GSA}\left({ }^{\circ}\right)$ & $8.3 \pm 7.2$ & $\ldots$ & $4.4 \pm 5.0$ & $<.001^{\mathrm{a}}$ \\
\hline
\end{tabular}

Abbreviations: SVA indicates sagittal vertical axis; PT, pelvic tilt; PI-LL, mismatch between pelvic incidence and lumbar lordosis; TK, thoracic kyphosis; SS, sacral slope; SFA, sacrofemoral angle; KA, knee angle; AA, ankle angle; PS, pelvic shift; GSA, global sagittal angle.

${ }^{\text {a } B o l d e d ~ v a l u e s ~ r e p r e s e n t ~ s t a t i s t i c a l l y ~ s i g n i f i c a n t ~ d i f f e r e n c e s ~ i n ~ p r e-~ a n d ~}$ postoperative alignment values to $P<.05$.

${ }^{b}$ Bolded values represent statistically significant differences between age-adjusted calculated ideal and actual postoperative alignment values to $P<.05$.

improved in alignment at early follow-up: $\Delta$ $\mathrm{SVA}=-37.3 \pm 59.0 \mathrm{~mm}, \Delta \mathrm{PT}=-3.1^{\circ} \pm 8.4^{\circ}, \Delta$ PI-LL $=-11.0^{\circ} \pm 16.0^{\circ}(P<.001$ all $)$. There was an overall reduction in pelvic shift $(38.8 \pm 47.8$ $\mathrm{mm}$ to $16.7 \pm 38.7 \mathrm{~mm}$ ) and knee flexion $\left(9.8^{\circ} \pm 9.2^{\circ}\right.$ to $\left.6.8^{\circ} \pm 8.4^{\circ}\right)$ accompanied by the surgical correction $(P<.001$ both cases $)$; postoperative hip extension, however, remained unchanged $(P=.577)$. The improvement in global standing alignment was reflected in a significant GSA decrease $\left(8.3^{\circ} \pm 7.2^{\circ}\right.$ to $4.4^{\circ} \pm 5.0^{\circ}$, $P<.001)$.

\section{Postoperative Correction Groups}

Following correction, there was low congruity of actual alignment with age-adjusted ideals, with $27.7 \%, 28.7 \%$, and $23.9 \%$ of patients matching age targets in SVA, PT, and PI-LL, respectively (Figure 3). Patients were more frequently undercorrected, compared to age-adjusted targets, for PT $(41.0 \%)$ and PI-LL (43.6\%). However, in the SVA group, $42.0 \%$ of patients were overcorrected. Mean spino-pelvic values in each postoperative age-alignment correction group differed, with the most severe malalignment observed in undercorrections (Table 2). Use of $3 \mathrm{CO}$ did not significantly affect resultant correction group $(P>.05)$.

\section{SVA Correction Analysis}

Each SVA correction group was similar for BMI, UIV, LIV, and levels fused (range: 9.3-10.6) $(P>$ 0.05 all). For SVA, undercorrected patients were younger than overcorrected patients $(55.2 \pm 19.0$

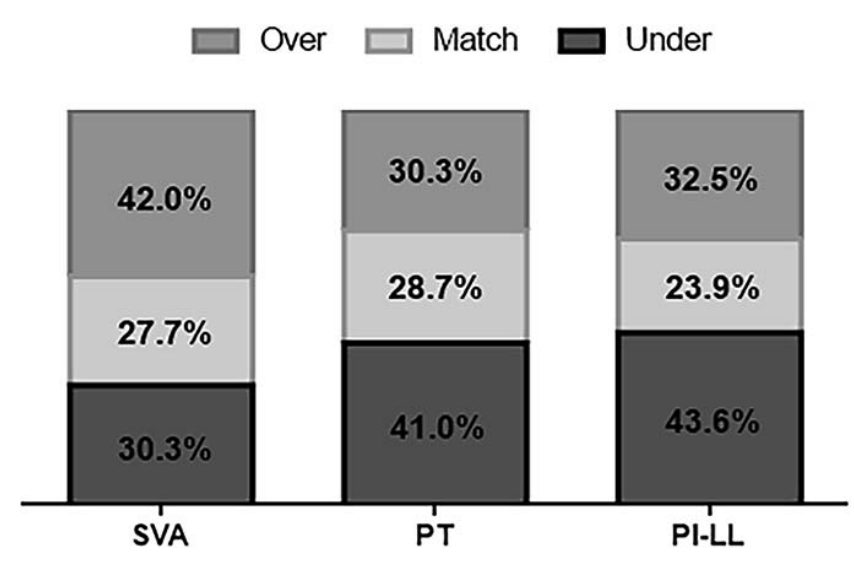

Figure 3. Rates of patients in each early postoperative spino-pelvic alignment group (Match, Undercorrect, Overcorrect) according to age-adjusted targets. Abbreviations: SVA, sagittal vertical axis; PI-LL, mismatch between pelvic incidence and lumbar lordosis; and PT, pelvic tilt.

vs. $68.6 \pm 8.7$ years, $P<.001)$. Undercorrected cases had the greatest offset between age-adjusted and actual postoperative alignment $(-61.6 \pm 32.0$ $\mathrm{mm})$. These patients showed the greatest pre- and postoperative anterior malalignment, resulting from the smallest correction (Table 2). Undercorrected SVA patients compensated for persistent postoperative anterior alignment with reduced TK $\left(40.9^{\circ} \pm 17.7^{\circ}\right)$ and increased KA $\left(9.0^{\circ} \pm 8.8^{\circ} ; P<.040\right.$ all; Table 3$)$. They also recruited an increased posterior pelvic shift $(50.5 \pm 36.9 \mathrm{~mm})$ compared with both matched and overcorrected cohorts $(P<.001$ all $)$. Undercorrection impacted full-body standing axis, displaying significantly larger GSA (undercorrected: $9.1^{\circ} \pm 5.2^{\circ}$, matched: $5.0^{\circ} \pm 3.3^{\circ}$, overcorrected: $\left.1.7^{\circ} \pm 3.3^{\circ}, P<.001\right)$.

\section{PT Correction Analysis}

The 3 pelvic tilt (PT) correction groups had similar age, UIV, LIV, and levels fused (range: 9.311.2 levels; $P>.05$ all). Age-undercorrected PT patients had a larger baseline BMI than overcorrections $\left(27.7 \pm 5.9\right.$ versus $24.3 \pm 4.9 \mathrm{~kg} / \mathrm{m}^{2}$, $P=.015)$. Undercorrected cases were on average $11^{\circ} \pm 7.3^{\circ}$ from age-adjusted goals. These patients displayed the largest baseline and postoperative PT, with the smallest degree of tilt change with surgery (Table 2). Undercorrections also reported a larger postoperative SVA than overcorrected cases (undercorrected: $88.7 \pm 72.3 \mathrm{~mm}$ versus overcorrected: $50.2 \pm 77.5 \mathrm{~mm}, P=.048)$. When patients were age-undercorrected for PT, they recruited more posterior pelvic shift $(37.7 \pm 32.7 \mathrm{~mm})$, hip extension $\left(212.4^{\circ} \pm 9.6^{\circ}\right)$, knee flexion, and ankle dorsi- 
Table 2. Actual measured spino-pelvic values for each age-adjusted alignment target group (Match, Undercorrect, Overcorrect) at baseline and early postoperative visit.

\begin{tabular}{|c|c|c|c|c|}
\hline $\begin{array}{l}\text { Postoperative } \\
\text { Groups }\end{array}$ & Under & Match & Over & $P$ \\
\hline \multicolumn{5}{|l|}{ SVA } \\
\hline Baseline & $105.7 \pm 69.8$ & $86.3 \pm 75.5$ & $47.4 \pm 63.1$ & $.002^{\mathrm{a}}$ \\
\hline Early postop & $87.0 \pm 50.0$ & $34.2 \pm 26.6$ & $1.0 \pm 27.6$ & $<.001^{\mathrm{a}}$ \\
\hline$\Delta$ & $-14.2 \pm 54.3$ & $-52.1 \pm 63.5$ & $-46.7 \pm 55.9$ & $.007^{\mathrm{a}}$ \\
\hline \multicolumn{5}{|l|}{ PT } \\
\hline Baseline & $33.9 \pm 11.3$ & $27.5 \pm 8.0$ & $17.6 \pm 9.6$ & $<.001^{\mathrm{a}}$ \\
\hline Early postop & $33.6 \pm 7.8$ & $22.2 \pm 4.5$ & $12.6 \pm 6.0$ & $.003^{\mathrm{a}}$ \\
\hline$\Delta$ & $-0.3 \pm 9 . .0$ & $-5.3 \pm 6.4$ & $-5.0 \pm 8.43$ & $<.001^{\mathrm{a}}$ \\
\hline \multicolumn{5}{|l|}{ PI-LL } \\
\hline Baseline & $26.2 \pm 23.1$ & $20.1 \pm 15.2$ & $5.4 \pm 21.3$ & $<.001^{\mathrm{a}}$ \\
\hline Early postop & $21.3 \pm 15.2$ & $8.2 \pm 6.6$ & $-8.7 \pm 9.1$ & $<.001^{\mathrm{a}}$ \\
\hline$\Delta$ & $-4.9 \pm 15.4$ & $-12.4 \pm 15.1$ & $-14.1 \pm 18.0$ & $.014^{\mathrm{a}}$ \\
\hline
\end{tabular}

Abbreviations: Under indicates undercorrect; Over, overcorrect; $\Delta$, change in parameter following surgery; SVA, sagittal vertical axis; PT, pelvic tilt; PI-LL, mismatch between pelvic incidence and lumbar lordosis.

${ }^{a}$ Bolded cells denote statistical significance across correction groups to $P<.05$.

flexion $(P<.044)$ to compensate (Table 4$)$; their GSA was consequently greater (undercorrected: $7.3^{\circ} \pm 4.9^{\circ}$, matched: $4.2^{\circ} \pm 4.6^{\circ}$, overcorrected: $\left.1.8^{\circ} \pm 4.3^{\circ}, P<.001\right)$.

\section{PI-LL Correction Analysis}

The UIV, LIV, and BMI for PI-LL age-adjusted correction groups were similar $(P>.05)$, though overcorrections underwent slightly longer fusions than matched cases (undercorrected: 9.9, matched:

Table 3. Adoption of compensatory mechanisms for SVA postoperative ageadjusted alignment groups (Match, Undercorrect, Overcorrect) at baseline and early follow-up.

\begin{tabular}{|c|c|c|c|c|}
\hline $\begin{array}{l}\text { SVA } \\
\text { Postoperative } \\
\text { Groups }\end{array}$ & $\begin{array}{c}\text { Under } \\
(38.2 . \%)\end{array}$ & $\begin{array}{c}\text { Match } \\
(25.2 \%)\end{array}$ & $\begin{array}{c}\text { Over } \\
(36.6 \%)\end{array}$ & $\boldsymbol{P}$ \\
\hline \multicolumn{5}{|l|}{$\mathrm{TK}$} \\
\hline Baseline & $34.7 \pm 20.8$ & $38.0 \pm 19.6$ & $41.7 \pm 18.7$ & .269 \\
\hline Early Postop & $40.9 \pm 17.7$ & $42.9 \pm 18.0$ & $49.9 \pm 15.6$ & $.028^{\mathrm{a}}$ \\
\hline$\Delta$ & $8.2 \pm 16.5$ & $2.9 \pm 16.1$ & $8.2 \pm 16.1$ & .269 \\
\hline \multicolumn{5}{|l|}{ SFA } \\
\hline Baseline & $203.4 \pm 9.9$ & $204.3 \pm 9.9$ & $206.7 \pm 8.0$ & .235 \\
\hline Early post-op & $203.4 \pm 12.1$ & $204.5 \pm 9.5$ & $205.1 \pm 9.5$ & .770 \\
\hline$\Delta$ & $1.5 \pm 7.0$ & $-0.2 \pm 6.8$ & $-1.9 \pm 6.2$ & .082 \\
\hline \multicolumn{5}{|l|}{$\mathrm{KA}$} \\
\hline Baseline & $11.9 \pm 10.3$ & $11.8 \pm 9.3$ & $8.4 \pm 7.5$ & .139 \\
\hline Early postop & $9.0 \pm 8.8$ & $8.6 \pm 8.1$ & $5.3 \pm 7.7$ & $.027^{\mathrm{a}}$ \\
\hline$\Delta$ & $-3.9 \pm 7.0$ & $-3.7 \pm 6.0$ & $-3.1 \pm 7.0$ & .844 \\
\hline \multicolumn{5}{|l|}{ AA } \\
\hline Baseline & $7.3 \pm 4.3$ & $8.3 \pm 3.9$ & $8.1 \pm 3.7$ & .528 \\
\hline Early postop & $6.0 \pm 5.3$ & $8.4 \pm 4.0$ & $7.9 \pm 3.8$ & .067 \\
\hline$\Delta$ & $-1.6 \pm 4.2$ & $0.0 \pm 4.2$ & $-0.2 \pm 3.2$ & .184 \\
\hline \multicolumn{5}{|l|}{ PS } \\
\hline Baseline & $59.1 \pm 39.7$ & $47.1 \pm 58.6$ & $27.0 \pm 36.8$ & $.008^{\mathrm{a}}$ \\
\hline Early postop & $50.5 \pm 36.8$ & $17.0 \pm 29.0$ & $-2.4 \pm 31.9$ & $<.001^{\mathrm{a}}$ \\
\hline$\Delta$ & $-10.0 \pm 44.0$ & $-34.5 \pm 46.3$ & $-29.4 \pm 33.9$ & $.026^{\mathrm{a}}$ \\
\hline
\end{tabular}

Abbreviations: Under indicates undercorrect; Over, overcorrect; $\Delta$, change in parameter following surgery; SVA, sagittal vertical axis; TK, thoracic kyphosis; SFA, sacrofemoral angle; KA, knee angle; AA, ankle angle; PS, pelvic shift.

a Bolded cells denote statistical significance between indicated correction groups to $P<.05$.
Table 4. Adoption of lower extremity compensatory mechanisms for PT postoperative age-adjusted alignment groups (Match, Undercorrect, Overcorrect) at baseline and early follow-up.

\begin{tabular}{|c|c|c|c|c|}
\hline $\begin{array}{l}\text { PT } \\
\text { Postoperative } \\
\text { Groups }\end{array}$ & $\begin{array}{c}\text { Under } \\
(41.6 \%)\end{array}$ & $\begin{array}{c}\text { Match } \\
(29.2 \%)\end{array}$ & $\begin{array}{c}\text { Over } \\
(29.2 \%)\end{array}$ & $P$ \\
\hline \multicolumn{5}{|l|}{ TK } \\
\hline Baseline & $38.4 \pm 17.0$ & $42.4 \pm 22.5$ & $35.2 \pm 20.7$ & .308 \\
\hline Early postop & $44.0 \pm 17.4$ & $47.8 \pm 14.5$ & $44.0 \pm 20.0$ & .554 \\
\hline$\Delta$ & $5.6 \pm 17.9$ & $5.4 \pm 16.5$ & $8.9 \pm 12.6$ & .570 \\
\hline \multicolumn{5}{|l|}{ SFA } \\
\hline Baseline & $209.5 \pm 9.6$ & $204.1 \pm 7.5$ & $198.8 \pm 7.6$ & $<.001^{\mathrm{a}}$ \\
\hline Early postop & $212.4 \pm 9.6$ & $204.1 \pm 7.5$ & $195.2 \pm 6.5$ & $<.001^{\mathrm{a}}$ \\
\hline$\Delta$ & $2.6 \pm 6.3$ & $-1.1 \pm 5.4$ & $-3.4 \pm 6.8$ & $<.001^{\mathrm{a}}$ \\
\hline \multicolumn{5}{|l|}{ KA } \\
\hline Baseline & $10.8 \pm 8.9$ & $12.6 \pm 9.3$ & $6.4 \pm 8.4$ & $.041^{\mathrm{a}}$ \\
\hline Early postop & $8.4 \pm 7.6$ & $8.5 \pm 9.2$ & $6.7 \pm 7.8$ & $.018^{a}$ \\
\hline$\Delta$ & $-2.7 \pm 6.8$ & $-4.8 \pm 6.1$ & $-3.2 \pm 6.2$ & .370 \\
\hline \multicolumn{5}{|l|}{$\mathrm{AA}$} \\
\hline Baseline & $7.8 \pm 4.0$ & $8.8 \pm 4.0$ & $6.9 \pm 3.9$ & .146 \\
\hline Early postop & $7.6 \pm 3.5$ & $8.6 \pm 4.2$ & $6.0 \pm 5.3$ & $.044^{\mathrm{a}}$ \\
\hline$\Delta$ & $-0.4 \pm 3.8$ & $-2.1 \pm 4.1$ & $-1.0 \pm 3.5$ & .694 \\
\hline \multicolumn{5}{|l|}{${ }^{\mathrm{a}} \mathrm{PS}$} \\
\hline Baseline & $53.7 \pm 45.6$ & $47.9 \pm 47.3$ & $13.9 \pm 40.6$ & $<.001^{\mathrm{a}}$ \\
\hline Early postop & $37.7 \pm 32.7$ & $11.7 \pm 33.1$ & $-4.6 \pm 37.6$ & $<.001^{\mathrm{a}}$ \\
\hline$\Delta$ & $-15.1 \pm 37.5$ & $-40.6 \pm 41.5$ & $-22.0 \pm 42.4$ & $.017^{\mathrm{a}}$ \\
\hline
\end{tabular}

Abbreviations: Under indicates undercorrect; Over, overcorrect; $\Delta$, change in parameter following surgery; PT, pelvic tilt; TK, thoracic kyphosis; SFA, sacrofemoral angle; KA, knee angle; AA, ankle angle; PS, pelvic shift.

${ }^{a}$ Bolded cells denote statistical significance between indicated correction groups to $P<.05$.

8.5, overcorrected: $11.1, P=.047)$. Undercorrected patients were younger than both the matched and overcorrected groups (undercorrected: $59.0 \pm 16.7$ years, matched: $65.9 \pm 11.1$ years, overcorrected: $65.5 \pm 10.4$ years, $P=.034)$. Patients undercorrected for PI-LL were on average $20.0^{\circ} \pm 13.2^{\circ}$ from the age-adjusted targets. Undercorrected patients had a significantly greater PI-LL than all correction groups at baseline and early postoperative visit (Table 2). The degree of correction in the age-undercorrected PI-LL group versus matched cases was smallest. At early follow-up, PI-LL undercorrections were characterized by a larger SVA $(54.5 \pm 54.5 \mathrm{~mm})$ and PT $\left(27.7^{\circ} \pm 11.3^{\circ}\right)$ compared to matched and overcorrections $(P<.001$ all $)$. The undercorrected cohort demonstrated a significantly increased posterior pelvic shift $(39.3 \pm 34.1)$ with reduced TK $(37.0 \pm 16.1$; $P<.001$ all; Table 5). This sagittal profile was reflected in an increased GSA (undercorrected: $7.3^{\circ} \pm 4.9^{\circ}$, matched: $4.3^{\circ} \pm 3.1^{\circ}$, overcorrected: $\left.1.9^{\circ} \pm 4.6^{\circ}, P<.001\right)$. PI-LL overcorrected patients also displayed a significantly greater SFA $\left(208.6^{\circ} \pm 10.1^{\circ}\right)$ and more anterior pelvic displacement $(-6.6 \pm 38.2 \mathrm{~mm})$ compared to those undercorrected. 
Table 5. Adoption of lower extremity compensatory mechanisms for PI-LL postoperative age-adjusted alignment groups (Match, Undercorrect, Overcorrect) at baseline and early follow-up.

\begin{tabular}{|c|c|c|c|c|}
\hline $\begin{array}{l}\text { PI-LL } \\
\text { Postoperative } \\
\text { Groups }\end{array}$ & $\begin{array}{c}\text { Under } \\
(41.6 \%)\end{array}$ & $\begin{array}{c}\text { Match } \\
(29.2 \%)\end{array}$ & $\begin{array}{c}\text { Over } \\
(29.2 \%)\end{array}$ & $P$ \\
\hline \multicolumn{5}{|l|}{ TK } \\
\hline Baseline & $31.1 \pm 18.8$ & $38.2 \pm 14.9$ & $49.3 \pm 20.1$ & $<.001^{\mathrm{a}}$ \\
\hline Early postop & $37.0 \pm 16.1$ & $45.7 \pm 16.2$ & $55.7 \pm 14.2$ & $<.001^{\mathrm{a}}$ \\
\hline$\Delta$ & $6.4 \pm 18.6$ & $7.5 \pm 13.0$ & $6.3 \pm 15.3$ & .950 \\
\hline \multicolumn{5}{|l|}{ SFA } \\
\hline Baseline & $202.0 \pm 8.3$ & $204.4 \pm 8.0$ & $208.8 \pm 10.0$ & $.011^{\mathrm{a}}$ \\
\hline Early postop & $198.5 \pm 8.8$ & $205.1 \pm 8.8$ & $208.6 \pm 10.1$ & $<.001^{\mathrm{a}}$ \\
\hline$\Delta$ & $-3.4 \pm 7.4$ & $0.2 \pm 5.9$ & $1.6 \pm 5.9^{\mathrm{a}}$ & $.003^{\mathrm{a}}$ \\
\hline \multicolumn{5}{|l|}{ KA } \\
\hline Baseline & $10.4 \pm 8.8$ & $10.9 \pm 8.3$ & $10.2 \pm 9.6$ & .950 \\
\hline Early postop & $7.9 \pm 8.0$ & $8.5 \pm 6.5$ & $5.6 \pm 9.6$ & .306 \\
\hline$\Delta$ & $-3.2 \pm 6.3$ & $-2.3 \pm 6.8$ & $-4.8 \pm 6.2$ & .328 \\
\hline \multicolumn{5}{|l|}{ AA } \\
\hline Baseline & $7.4 \pm 3.7$ & $8.2 \pm 3.9$ & $8.5 \pm 4.2$ & .472 \\
\hline Early postop & $6.9 \pm 3.4$ & $8.9 \pm 3.1$ & $7.4 \pm 5.9$ & .144 \\
\hline$\Delta$ & $-0.8 \pm 3.5$ & $0.8 \pm 3.7$ & $-1.0 \pm 4.2$ & .150 \\
\hline \multicolumn{5}{|l|}{ PS } \\
\hline Baseline & $53.6 \pm 45.5$ & $44.3 \pm 34.6$ & $26.1 \pm 52.1$ & $.028^{\mathrm{a}}$ \\
\hline Early postop & $39.3 \pm 34.1$ & $14.0 \pm 26.6$ & $-6.6 \pm 38.2$ & $<.001^{\mathrm{a}}$ \\
\hline$\Delta$ & $-15.9 \pm 36.6$ & $-30.8 \pm 32.3$ & $-34.9 \pm 50.8$ & .103 \\
\hline
\end{tabular}

Abbreviations: Under indicates undercorrect; Over, overcorrect; $\Delta$, change in parameter following surgery; PI-LL, mismatch between pelvic incidence and lumbar lordosis; PT, pelvic tilt; TK, thoracic kyphosis; SFA, sacrofemoral angle; KA, knee angle; AA, ankle angle; PS, pelvic shift.

${ }^{a}$ Bolded cells denote statistical significance between indicated correction groups to $P<.05$.

\section{Effect of Lumbosacral Fusion or Pelvic Fixation on Lower Limb Compensation}

There were $79(65 \%)$ patients with fusion to lumbosacral region or pelvic fixation. Patients with lumbosacral fusions/sacrum/pelvic fixation experienced a larger decrease in KA from pre- to postoperatively than patients without lumbosacral fusions or pelvic fixation (with: $-4.2^{\circ}$, without: $-0.6^{\circ}, P=.003$ ). Pelvic shift significantly decreased postoperatively for patients with lumbosacral fusions or pelvic fixation and increased for patients without (with: $-36.8 \mathrm{~mm}$, without: $+10.7 \mathrm{~mm}$, $P<.001)$. GSA also decreased for patients with lumbosacral fusions and/or pelvic fixation and increased for patients without (with: $-5.4^{\circ}$, without: $\left.+0.48^{\circ}, P<.001\right)$. Patients with lumbosacral fusion or pelvic fixation did not show different changes in SFA and AA pre- to postoperatively (both $P>.05)$.

\section{Relationship between Radiographic Correction and Clinical Outcomes}

In looking at SVA alignment groups, we assessed clinical scores for the Oswestry Disability index (ODI), neck disability index (NDI), and visual analog scale (VAS) neck, arm, back, and leg pain scores for patients who were matched for SVA, undercorrected, and overcorrected. Patients who matched their target SVA trended toward improvement in VAS back pain scores (preop: $6.1 \pm 3$, postop: $4.1 \pm 2.3, P=.056)$. Patients who were undercorrected with respect to SVA worsened in ODI (preop: $40 \pm 26.2$, postop: $53.8 \pm 20.1$, $P=.038$ ) and NDI (preop: $31.3 \pm 23.4$, postop: $48.5 \pm 16.6, P=.0009)$ after surgery. Patients who were overcorrected with respect to SVA displayed improvements in VAS neck and leg pain scores.

\section{Alignment Sustainability}

A subanalysis of patients with 1-year postoperative radiographs (in addition to the 6-month followup for the entire cohort) was performed. The rates of patients reaching age-adjusted threshold ranges by 1 year postoperative were as follows:

- SVA: undercorrected to matched $=0.0 \%$; overcorrected to matched $=28.6 \%$

- PT: undercorrected to matched $=26.7 \%$; overcorrected to matched $=31.2 \%$

- PI-LL: undercorrected to matched $=15.4 \%$; overcorrected to matched $=14.3 \%$

Matches in all spino-pelvic age-adjusted correction groups maintained optimal alignment $(P>.05)$. Undercorrections showed significant changes in compensation from early to 1-year follow-up visits in this subset of patients (Table 6): undercorrection for SVA displayed a decrease in TK $\left(\Delta-7.8^{\circ}, P=.032\right)$ with more anterior PS $(\Delta-13.2$ $\mathrm{mm}, P=.039)$; undercorrection for PT decreased hip extension $\left(\Delta-3.7^{\circ}, P=.004\right)$ and TK $\left(\Delta-4.7^{\circ}\right.$, $P=.036)$; undercorrection for PI-LL only resulted in significant increase in TK $\left(\Delta 6.3^{\circ}, P=.009\right)$.

\section{DISCUSSION}

Full-body imaging is a powerful tool for extensive visualization and analysis of sagittal alignment in treating complex spinal deformity. However, these principles remain untested in a postoperative setting, wherein compensation may be required to offset unfavorable realignment. There have been a number of articles previously published on sagittal balance of the mobile spine, but fewer on ageadjusted alignment targets in spine surgery. ${ }^{22,23}$ To our knowledge, the only studies describing full-body postoperative alignment are in hip and acetabular pathology, without characterization of lower ex- 
Table 6. Changes in sagittal alignment from early $(\leq 6 \mathrm{M})$ to 1 -year postoperative visit for each spino-pelvic age-adjusted correction group (Match, Undercorrect, Overcorrect)

\begin{tabular}{|c|c|c|c|c|c|c|c|c|}
\hline Postoperative Correction Groups & $\triangle \mathrm{SVA}(\mathrm{mm})$ & $\Delta$ PT $\left({ }^{\circ}\right)$ & $\Delta \mathbf{L L}\left({ }^{\circ}\right)$ & $\Delta$ TK $\left(^{\circ}\right)$ & $\Delta$ SFA $\left({ }^{\circ}\right)$ & $\Delta \mathbf{K A}\left({ }^{\circ}\right)$ & $\Delta \mathbf{A A}\left({ }^{\circ}\right)$ & $\Delta$ PS (mm) \\
\hline \multicolumn{9}{|l|}{ SVA } \\
\hline Under & -0.1 & -2.5 & 0.3 & $-7.8^{\mathrm{a}}$ & -1.4 & -2.0 & -0.9 & $-13.2^{\mathrm{a}}$ \\
\hline Match & -1.4 & 0.7 & 0.3 & -1.7 & -1.5 & 2.0 & -0.2 & 12.6 \\
\hline Over & $15.6^{\mathrm{a}}$ & 1.5 & -2.5 & 1.2 & -0.7 & $2.0^{\mathrm{a}}$ & -0.1 & 11.3 \\
\hline \multicolumn{9}{|l|}{ PT } \\
\hline Under & 11.6 & -1.8 & -1.3 & -4.7 & $-3.7^{\mathrm{a}}$ & 2.8 & 0.9 & -0.0 \\
\hline Match & 5.2 & 1.5 & 1.2 & 2.8 & 1.4 & -1.0 & -1.1 & 2.3 \\
\hline Over & 6.6 & 2.1 & $-2.9^{\mathrm{a}}$ & 0.7 & 0.6 & 0.5 & -0.9 & 13.7 \\
\hline \multicolumn{9}{|l|}{ PI-LL } \\
\hline Under & 10.4 & -1.9 & -0.1 & $6.3^{\mathrm{a}}$ & -2.9 & 1.3 & 0.3 & -4.2 \\
\hline Match & 10.0 & 0.3 & -1.0 & 0.0 & -2.1 & 11.1 & 0.2 & 10.7 \\
\hline Over & 4.8 & 2.6 & -2.4 & 1.3 & 1.3 & -0.4 & -1.2 & 11.3 \\
\hline
\end{tabular}

Abbreviations: Under indicates undercorrect; Over, overcorrect; $\Delta$, change in parameter following surgery; SVA, sagittal vertical axis; PT, pelvic tilt; PI-LL, mismatch between pelvic incidence and lumbar lordosis; TK, thoracic kyphosis; SFA, sacrofemoral angle; KA, knee angle; AA, ankle angle; PS, pelvic shift.

${ }^{a}$ Bolded cells denote a statistically significant change to $P<.05$ in alignment value from early to l-year follow-up visits.

tremity alignment changes. ${ }^{24,25}$ This study presents a pre- and postoperative investigation of variations in sagittal alignment and compensation based on deviations from age-adjusted deformity thresholds for optimal correction.

Expectedly, patients displayed significant improvements in spino-pelvic parameters at the early postoperative visit. Optimal global postoperative alignment assessment demands analysis of the lower extremities, as these mechanisms work to regulate standing posture. ${ }^{16,26,27}$ Consistent with the overall SVA decrease and lordosis restoration, the cohort's pelvic displacement shifted anteriorly $(\Delta-24.0 \mathrm{~mm})$ coupled with reduction of knee flexion $\left(\Delta-3.3^{\circ}\right)$. This lower-limb decompensation follows sequential compensatory mechanism recruitment and relaxation given improved spinal alignment to maintain erect posture. ${ }^{16}$ The cohort's decrease in GSA, accounting for simultaneous spino-pelvic deformity and lower-limb compensation, confirms this fullbody change. ${ }^{20}$

Failure to realign deformity patients frequently results in hardware complications and poor longterm outcomes.9,28,29 Surgical plans built on ageadjusted targets, providing clinically relevant patient-specific thresholds, are essential to mitigate this risk. $^{8}$ In the current study, by applying these ageadjusted ideals for classic spino-pelvic measures, we were able to determine rates of success and failure following deformity correction. Interestingly, rates of patients' postoperative sagittal profile matching a \pm 10 -year age range were lower than prior reports evaluating successful versus failed ASD procedures. Against PT and PI-LL age ideals, patients tended to display high rates of undercorrection; only for SVA did patients reach a higher success (match or overcorrection) rate $(69.7 \%)$, which compares well to the $77 \%$ frequency proposed by Schwab et al. ${ }^{9}$ However, this study reveals that prior literature, basing alignment failures off of generalized thresholds instead of an individualized goal, may underestimate actual undercorrection occurrence, particularly when considering the magnitude of deformity at baseline.

All undercorrections showed consistently larger baseline sagittal malalignment for each respective spino-pelvic parameter. Comparably, Lafage et al ${ }^{10}$ presented a $22 \%$ failure rate following thoracic $3 \mathrm{CO}$ and noted that these patients also displayed greater preoperative SVA, PT, and LL malalignment. Our findings are similar to the failure analysis done by Schwab et al of 79 ASD patients following 3CO: failed patients at $<6$-month postoperative followup had a significantly larger preoperative SVA, PT, and PI than successful realignments. ${ }^{9}$ Collectively, these results support the concept that optimal realignment requires a specific degree of correction that proportionally matches a single patient's baseline deformity, which may be prospectively achieved via age-adjusted correction formulas. For SVA and PI-LL, undercorrected patients were younger (SVA: undercorrected: 55.2 years versus overcorrected: 68.6 years; PI-LL: undercorrected: 59.0 years, matched: 65.9 years, overcorrected: 65.5 years) than successful realignments, suggesting that despite older ASD cases requiring less rigorous ageidealized thresholds, greater baseline deformity in undercorrections was the predominant driver of inferior radiographic outcomes. ${ }^{8}$ Effectively, despite undergoing comparable fusions, undercorrections displayed the smallest amount of regional correction 
per spino-pelvic parameter considered against all other groups.

SVA, PT, PI-LL mismatch present with variable severity for each patient, and may differentially hinder the ability to compensate throughout the spine or lower limbs. As seen in our analysis according to each parameter, deformities with different malalignment as measured by these characteristics triggered different methods for compensation.

At early postoperative visit, SVA undercorrection to age-adjusted ideals, marked by a mean offset of $62 \mathrm{~mm}$, correlated with increasing posterior pelvic shift, flexed knees, and thoracic hypokyphosis, all well-documented mechanisms to compensate for an anterior displacement of C7. ${ }^{16,30}$ At 1-year followup, undercorrected SVA patients were unable to regain age-ideal alignment $(0.0 \%$ undercorrectionto-match), despite apparent thoracic flattening and anterior pelvic displacement. Though the contribution of TK to counteracting excessive anterior malalignment has been described, it is possible that these patients did not retain the flexibility required; extension of fusion to cephalad levels may further reduce this possibility, though the UIV of SVA correction groups was similar. ${ }^{31,32}$

When PT was undercorrected, knee flexion and pelvic shift were adopted, consistent with prior reports theorizing the transfer of compensation to lower limbs when pelvis retroversion is exhausted. ${ }^{15,16,33}$ Patients at risk for PT undercorrection based on age-adjusted targets displayed the largest preoperative tilt and highest $\mathrm{BMI}$, factors also implicated in ASD failures analyzed by Schwab et al. ${ }^{9}$ These same patients displayed PT normalization 1 year postoperation via a reduction in hip extension (SFA $\Delta-3.7^{\circ}$ ) and thoracic compensation $\left(\Delta-4.7^{\circ}\right)$.

Patients undercorrected to age-adjusted PI-LL thresholds were distinct in smaller postoperative TK and greater pelvic shift to mitigate the residual spino-pelvic mismatch and anterior malalignment $\left(\mathrm{SVA}=55 \mathrm{~mm}, \mathrm{PT}=28^{\circ}\right)$. This hypokyphotic thoracic posture with posterior pelvic displacement assumed for maximum compensation with positive SVA and inadequate LL has been described. ${ }^{15,34,35}$ Moreover, patients overcorrected in PI-LL displayed a comparable profile as previously described by Ferrero et $\mathrm{al}^{16}$ : heightened hip extension and thoracic kyphosis, with anterior pelvic displacement acting as compensation, with overall neutral global alignment and low PT ${ }^{16}$ Consistent with the smaller PI-LL correction $\left(\Delta-4.5^{\circ}\right)$ in age-undercorrected patients, Jang et al noted a strong correlation between the lumbar correction angle and the spontaneous postoperative thoracic curve change. ${ }^{36}$ Subsequent rapid exhaustion of the hypokyphotic compensatory mechanisms was also noted by 1-year follow-up in undercorrected cases, with a significant increase in TK $\left(\Delta 6.3^{\circ}\right)$. Prior series have similarly noted kyphotic instability and progression following insufficient LL correction: Lafage et $\mathrm{al}^{31}$ reported on 18 patients with unfavorable postoperative TK after pedicle subtraction osteotomy, which was theoretically driven by inadequate postoperative LL restoration. Interestingly though, no supplemental variations in lower extremity compensation were noted in age-undercorrected PI-LL patients at 1 year postoperation.

\section{Limitations}

A primary limitation of this study is the heterogeneous nature of spinal deformity, which was not controlled for beyond fusion extent. Further, local compensatory mechanisms of retrolisthesis and adjacent segment hyperextension were not evaluated. In addition, the EOS database is currently limited in the evaluation of intrinsic joint disease that might influence the position of the hips, knees, and ankles in compensatory mechanisms. This study is lacking coronal imbalance assessment that likely contributes to clinical symptoms that should be investigated further. As this was primarily a radiographic study, correlations between clinical outcome measures and observed compensatory mechanisms should be subsequently evaluated with extended postoperative follow-up.

\section{Conclusion}

This is the first study to analyze full-body alignment following spinal deformity correction using novel age-adjusted formulas for radiographic thresholds as a benchmark for surgical success. Undercorrection is underappreciated using traditional standardized thresholds. Differential compensation recruited in undercorrections according to spino-pelvic parameter highlights the dynamic relationship between spinal regions and certain musculoskeletal components in patient-specific deformity evaluation. 


\section{REFERENCES}

1. Glassman SD, Bridwell KM, Dimar JR, Horton W, Berven $\mathrm{S}$, Schwab F. The impact of positive sagittal balance in adult spinal deformity. Spine (Phila Pa 1976). 2005;30(18):2024-2029. https://doi.org/10.1097/01.brs.0000179086.30449.96.

2. Glassman SD, Berven S, Bridwell K, Horton W, Dimar JR. Correlation of radiographic parameters and clinical symptoms in adult scoliosis. Spine (Phila Pa 1976). 2005;30(6):682-688. https://doi.org/10.1097/01.brs.0000155425. 04536.f7.

3. Lafage V, Schwab FJ, Patel A, Hawkinson N, Farcy J-P. Pelvic tilt and truncal inclination: two key radiographic parameters in the setting of adults with spinal deformity. Spine (Phila Pa 1976). 2009;34(17):E599-E606. https://doi.org/10. 1097/BRS.0b013e3181aad219.

4. Schwab FJ, Blondel B, Bess S, et al. Radiographical spinopelvic parameters and disability in the setting of adult spinal deformity: a prospective multicenter analysis. Spine (Phila Pa 1976). 2013;38(13):E803-E812. https://doi.org/10. 1097/BRS.0b013e318292b7b9.

5. Bridwell KH, Glassman $\mathrm{S}$, Horton $\mathrm{W}$, et al. Does treatment (nonoperative and operative) improve the two-year quality of life in patients with adult symptomatic lumbar scoliosis: a prospective multicenter evidence-based medicine study. Spine (Phila Pa 1976). 2009;34(20):2171-2178. https:// doi.org/10.1097/BRS.0b013e3181a8fdc8.

6. Smith JS, Shaffrey CI, Berven S, et al. Operative versus nonoperative treatment of leg pain in adults with scoliosis: a retrospective review of a prospective multicenter database with two-year follow-up. Spine (Phila Pa 1976). 2009;34(16):16931698. https://doi.org/10.1097/BRS.0b013e3181ac5fcd.

7. Schwab F, Ungar B, Blondel B, et al. Scoliosis Research Society-Schwab adult spinal deformity classification: a validation study. Spine (Phila Pa 1976). 2012;37(12):1077-1082. https://doi.org/10.1097/BRS.0b013e31823e15e2.

8. Lafage R, Schwab F, Challier V, et al. Defining spino-pelvic alignment thresholds: should operative goals in adult spinal deformity surgery account for age? Spine (Phila Pa 1976). 2016;41(1):62-68. https://doi.org/10.1097/BRS.0000000000001171.

9. Schwab FJ, Patel A, Shaffrey CI, et al. Sagittal realignment failures following pedicle subtraction osteotomy surgery: are we doing enough?: Clinical article. J Neurosurg Spine. 2012;16(6):539-546. https://doi.org/10.3171/2012.2. SPINE11120.

10. Lafage V, Smith JS, Bess S, et al. Sagittal spino-pelvic alignment failures following three column thoracic osteotomy for adult spinal deformity. Eur Spine J. 2012;21(4):698-704. https://doi.org/10.1007/s00586-011-1967-3.

11. Gilad R, Gandhi CD, Arginteanu MS, Moore FM, Steinberger A, Camins M. Uncorrected sagittal plane imbalance predisposes to symptomatic instrumentation failure. Spine J. 2008;8(6):911-917. https://doi.org/10.1016/j.spinee.2007.10. 035 .

12. Yagi M, Akilah KB, Boachie-Adjei O. Incidence, risk factors and classification of proximal junctional kyphosis: surgical outcomes review of adult idiopathic scoliosis. Spine (Phila Pa 1976). 2011;36(1):E60-E68. https://doi.org/10.1097/ BRS.0b013e3181eeaee2.

13. Jalai CM, Cruz DL, Diebo BG, et al. Full-body analysis of age-adjusted alignment in adult spinal deformity patients and lower-limb compensation. Spine (Phila Pa 1976). 2017;42(9):653661. https://doi.org/10.1097/BRS.0000000000001863.

14. Vira S, Diebo BG, Spiegel MA, et al. Is there a genderspecific recruitment pattern in the setting of progressive sagittal malalignment? In: The 22nd International Meeting on Advanced Spine Techniques (IMAST). Kuala Lumpur, Malaysia; 2015.

15. Diebo BG, Ferrero E, Lafage R, et al. Recruitment of compensatory mechanisms in sagittal spinal malalignment is age and regional deformity dependent: a full-standing axis analysis of key radiographical parameters. Spine (Phila $\mathrm{Pa}$ 1976). 2015;40(9):642-649. https://doi.org/10.1097/BRS. 0000000000000844 .

16. Ferrero E, Liabaud B, Challier V, et al. Role of pelvic translation and lower-extremity compensation to maintain gravity line position in spinal deformity. J Neurosurg Spine. 2015;24(3):1-11. https://doi.org/10.3171/2015.5.SPINE14989.

17. Deschênes S, Charron G, Beaudoin G, et al. Diagnostic imaging of spinal deformities: reducing patients radiation dose with a new slot-scanning X-ray imager. Spine (Phila Pa 1976). 2010;35(9):989-994. https://doi.org/10.1097/BRS.0b013e3181bd caa4.

18. Rillardon L, Levassor N, Guigui P, et al. Validation of a tool to measure pelvic and spinal parameters of sagittal balance [in French]. Rev Chir Orthop Reparatrice Appar Mot. 2003;89(3):218-227.

19. Champain S, Benchikh K, NogierA, Mazel C, Guise J De, Skalli W. Validation of new clinical quantitative analysis software applicable in spine orthopaedic studies. Eur Spine J. 2006;15(6):982-991. https://doi.org/10.1007/s00586-005-0927-1.

20. Diebo BG, Oren JH, Challier V, et al. Global sagittal axis: a step toward full-body assessment of sagittal plane deformity in the human body. J Neurosurg Spine. 2016;25(4):16. https://doi.org/10.3171/2016.2.SPINE151311.

21. Mangione P, Sénégas J. Sagittal balance of the spine [in French]. Rev Chir Orthop Reparatrice Appar Mot. 1997;83(1):22-32.

22. Roussouly P, Pinheiro-Franco JL. Sagittal parameters of the spine: biomechanical approach. Eur Spine J. 2011;20(Suppl 5):578-585.

23. Le Huec J-CC, Aunoble S, Philippe L, Nicolas P. Pelvic parameters: origin and significance. Eur Spine J. 2011;20 Suppl 5(Suppl 5):564-571. https://doi.org/10.1007/s00586-011-1940-1.

24. Barbier O, Skalli W, Mainard L, Mainard D. The reliability of the anterior pelvic plane for computer navigated acetabular component placement during total hip arthroplasty: prospective study with the EOS imaging system. Orthop Traumatol Surg Res. 2014;100(6 Suppl):S287-S291. https:// doi.org/10.1016/j.otsr.2014.07.003.

25. Masquefa $\mathrm{T}$, Verdier $\mathrm{N}$, Gille $\mathrm{O}$, et al. Change in acetabular version after lumbar pedicle subtraction osteotomy to correct post-operative flat back: EOS measurements of 38 acetabula. Orthop Traumatol Surg Res. 2015;101(6):655-659. https://doi.org/10.1016/j.otsr.2015.07.013.

26. Lafage V, Schwab FJ, Skalli W, et al. Standing balance and sagittal plane spinal deformity: analysis of spinopelvic and gravity line parameters. Spine (Phila Pa 1976). 2008;33(14):15721578. https://doi.org/10.1097/BRS.0b013e31817886a2.

27. Obeid I, Hauger O, Aunoble SS, Bourghli A, Pellet N, Vital J-M. Global analysis of sagittal spinal alignment in major deformities: correlation between lack of lumbar lordosis and 
flexion of the knee. Eur Spine J. 2011;20(Suppl 5):681-685. https://doi.org/10.1007/s00586-011-1936-x.

28. Moal B, Schwab FJ, Ames CP, et al. Radiographic outcomes of adult spinal deformity correction: a critical analysis of variability and failures across deformity patterns. Spine Deform. 2014;2(3):219-225. https://doi.org/10.1016/j. jspd.2014.01.003.

29. Diebo BG, Henry J, Lafage V, Berjano P. Sagittal deformities of the spine: factors influencing the outcomes and complications. Eur Spine J. 2015;24(1):3-15. https://doi.org/10. 1007/s00586-014-3653-8.

30. Schwab FJ, Lafage V, Boyce R, Skalli W, Farcy J-PP. Gravity line analysis in adult volunteers: age-related correlation with spinal parameters, pelvic parameters, and foot position. Spine (Phila Pa 1976). 2006;31(25):E959-E967. https://doi.org/ 10.1097/01.brs.0000248126.96737.0f.

31. Lafage V, Ames C, Schwab FJ, et al. Changes in thoracic kyphosis negatively impact sagittal alignment after lumbar pedicle subtraction osteotomy: a comprehensive radiographic analysis. Spine (Phila Pa 1976). 2012;37(3):E180-E187. https:// doi.org/10.1097/BRS.0b013e318225b926.

32. Fujimori $\mathrm{T}$, Inoue $\mathrm{S}$, Le $\mathrm{H}$, et al. Long fusion from sacrum to thoracic spine for adult spinal deformity with sagittal imbalance: upper versus lower thoracic spine as site of upper instrumented vertebra. Neurosurg Focus. 2014;36(5):E9. https:// doi.org/10.3171/2014.3.FOCUS13541.

33. Bs SL, Lafage V, Ferrero E, et al. Chain of compensation related to PI-LL mismatch: a complete standing axis investigation including lower extremities. 2014;14(11):S75. https://doi. org/10.1016/j.spinee.2014.08.191.

34. Fletcher ND, Hopkins J, McClung A, Browne R, Sucato DJ. Residual thoracic hypokyphosis after posterior spinal fusion and instrumentation in adolescent idiopathic scoliosis: risk factors and clinical ramifications. Spine (Phila Pa 1976). 2012;37(3):200206. https://doi.org/10.1097/BRS.0b013e318216106c.

35. Obeid I, Boniello A, Boissiere L, et al. Cervical spine alignment following lumbar pedicle subtraction osteotomy for sagittal imbalance. Eur Spine J. 2015;23(Suppl 6):644-649. https://doi.org/10.1007/s00586-014-3738-4.

36. Jang J-S, Lee S-H, Min J-H, Maeng DH. Influence of lumbar lordosis restoration on thoracic curve and sagittal position in lumbar degenerative kyphosis patients. Spine (Phila Pa 1976). 2009;34(3):280-284. https://doi.org/10.1097/BRS. 0b013e318191e792.

Disclosures and COI: The authors report no conflicts of interest concerning the materials or methods used in this study or the findings specified in this paper. Institutional Review broad approval was obtained prior to study.

Corresponding Author: Peter G Passias, MD, New York Spine Institute, New York University Medical Center-Hospital for Joint Diseases, 301 East 17th St, New York, NY 10003. Phone: (516) 357-8777; Fax: (516) 357-0087; Email: peter. passias@nyumc.org.

Published 30 April 2019

This manuscript is generously published free of charge by ISASS, the International Society for the Advancement of Spine Surgery. Copyright (C) 2019 ISASS. To see more or order reprints or permissions, see http://ijssurgery.com. 